\title{
Sobre alguns aspectos metodológicos de pes- quisas em movimentação dentária induzida e das reabsorções dentárias: uma proposta de guia e cuidados para análise de trabalhos
}

\author{
Alberto Consolaro*
}

Resumo

Uma das principais características da metodologia científica está na descrição detalhada de todas as condições experimentais e especialmente dos critérios adotados para se constituir uma amostra e obterem-se os dados. Esta característica deve ser aplicada em todos os trabalhos para permitir a outros estudiosos repetir o mesmo trabalho sob as mesmas condições descritas, e assim obter-se os mesmos resultados em qualquer parte do mundo. Esta possibilidade, além de permitir a checagem dos dados relatados, confere uma maior credibilidade perante a comunidade científica. Em ciência os dados devem ser públicos e reprodutíveis. Ao estudar-se as reabsorções dentárias, a padronização da amostra, dos critérios de diagnóstico e de classificação é muito importante. A reabsorção dentária representa um fenômeno multifatorial, ou seja, pode ser induzida por muitas causas e influenciadas por inúmeros fatores como morfologia dentária e óssea, proporção coroa-raiz, tipo de má oclusão, técnica empregada, extensão do movimento e outros. Para um trabalho sobre este tema apresentar maior credibilidade em seus resultados requer-se uma amostra muito homogênea, uma padronização do diagnóstico e da técnica ortodôntica com alto nível de calibração. Infelizmente muitos trabalhos sobre reabsorções dentárias, especialmente as que ocorrem durante o tratamento ortodôntico, são estudadas em amostras acumuladas no tempo, sem padronização diagnóstica e técnica, com pacientes tratados por inúmeros operadores, sem considerar a necessidade de padronização da amostra em algumas variáveis importantes nem tampouco relevá-las ou discuti-las quando das análises dos resultados. O objetivo deste trabalho constitui induzir reflexões sobre este assunto e sugerir alguns parâmetros para a análise de trabalhos de pesquisas pertinentes às reabsorções dentárias e à biologia da movimentação ortodôntica.

Palavras-chave: Reabsorções dentárias. Movimentação ortodôntica. Metodologia de pesquisa.

* Professor Titular em Patologia da Faculdade de Odontologia de Bauru, USP. 


\section{INTRODUÇÃO}

Ao analisarmos um trabalho científico devemos lembrar dos ensinamentos de John Hunter (17281793) e de um de seus princípios: "Don't think, try the experiment" ou "Não ache, faça um experimento". Em outras palavras, Charles Dickens (1812-1870) expressou o mesmo pensamento: "Não aceite nada pela aparência, mas apenas pela evidência" (Enciclopédia Britânica).

Sir John Hunter foi um cirurgião escocês reconhecido como o pioneiro da cirurgia experimental médica e dentária e quem formulou a primeira explicação biológica para o movimento ortodôntico. Preconizou no futuro movimentar os dentes mal posicionados em vez de extraí-los. Em 1778 observou que frente a forças aplicadas o osso aumentava em espessura por adição nas suas superfícies externas, enquanto na porção interna era removida quantidade proporcional. Hunter também descreveu os fenômenos de reabsorção radicular após o transplante de dentes em humanos, bem como atribuiu nomes a cada um dos dentes, nomes que permanecem até hoje.

Esta abordagem sobre metodologia científca tem como objetivo singelo servir de guia para analisar-se um trabalho científico na área da Movimentação Dentária Induzida e das Reabsorções Dentárias em Ortodontia. Trata-se de um guia para ajudar na escolha de trabalhos de pesquisas na área que devam ou não servir de referência para comparações e parâmetros nas teses, dissertações, seminários e aulas. Se este objetivo for cumprido, que sirva de base para a adoção de condutas e protocolos clínicos em nossas futuras publicações.

\section{Premissa Inicial}

Imaginemos uma situação em que algum pesquisador resolva detectar o índice de reabsorção dentária em movimentos dentários induzidos em uma amostra de 120 pacientes ou então comparar este índice entre 3 técnicas diferentes, dividindoos em grupos, como por exemplo:

- Grupo de 40 pacientes submetidos à técnica de Edgewise Simplificada;

- Grupo de 40 pacientes submetidos à técnica de Straight Wire;

- Grupo de 40 pacientes submetidos à técnica da terapia Bioprogressiva.

Para isto, o pesquisador deve-se preocupar com alguns aspectos metodológicos muito importantes para que seus resultados e conclusões sejam verdadeiramente científicos. $\mathrm{O}$ dado científico deve ser metódico, passível de repetição e público em todas as suas nuances. Se não for possível, em função de sua grande heterogeneidade da amostra, cai por terra o caráter científico dos resultados do trabalho.

\section{Necessidade de grupo controle}

$\mathrm{Na}$ situação imaginada anteriormente torna-se importante observarmos mais 30 pacientes que nunca se submeteram a nenhum tratamento ortodôntico e acompanhá-los pelo mesmo período de tempo médio do tratamento. Estes pacientes devem ter o mesmo perfil sócio-econômico, considerar ainda a mesma faixa etária e o mesmo número de pacientes conforme o gênero.

Para que este tipo de cuidado?

Para descartar a possibilidade de que as reabsorções dentárias encontradas durante o tratamento não sejam decorrentes do uso normal dos dentes durante um certo período de vida do paciente.

Um argumento contrário à instalação deste grupo seria: mas os pacientes não serão radiografados antes do tratamento?

Sim, mas o período de tempo de vida transtratamento requer acompanhamento simultâneo. As reabsorções podem ser fruto de traumatismos, vícios e outros aspectos próprios da faixa etária e do gênero.

\section{Cuidados durante a amostragem: uniformi- dade da amostra}

Como foram escolhidos os pacientes? Quais são os critérios de escolha? Como foi a amostragem? Amostragem corresponde ao processo pelo 
qual se obtém uma determinada amostra; amostra por sua vez constitui uma parte representativa do universo sobre a qual aplica-se critérios e métodos experimentais.

Há uma exigência cada vez maior de casuísticas acumuladas no tempo não serem consideradas. Hoje, valoriza-se cada vez mais a casuística planejada e obtida simultaneamente em período de tempo predeterminado e planejado.

Este tipo de cuidado leva em consideração que todos os pacientes do grupo serão submetidos aos mesmos fatores temporais, sociais, econômicos e culturais. As casuísticas acumuladas ao longo do tempo tendem a ser heterogêneas em função de diferentes operadores, cuidados e fatores técnicos diferentes em épocas diferentes, muitos dados serão baseados em registros escritos nem sempre devidamente detalhados e fiéis.

Um dos critérios corresponde ao sorteio entre muitas pessoas aptas a participar do estudo e para integrar um ou outro grupo. Outro critério que pode ser utilizado é a ordem rígida de chegada não planejada.

$\mathrm{Na}$ escolha por sorteio e chegada aleatória, os pacientes devem apresentar o perfil sócio-econômico, idade, gênero e outras características previamente estabelecidas.

Uma outra forma de escolher em um certo número de pacientes constitui sortear um número determinado entre os existentes. Por exemplo, escolher 40 pacientes com características tais entre 100 acumulados com o tempo e padronizados com estas características. O sorteio deve ser totalmente aleatório, randomizado, ou seja, casualizado, ou melhor, por acaso. Esta forma de montar uma amostra diminui o risco de vícios amostrais.

\section{Padronização do tipo de má oclusão}

Considerando o tipo de oclusão, muito importante no tratamento ortodôntico, para uma amostragem homogênea e para que os tipos de tratamento variáveis não interfiram nos resultados, os grupos deveriam ter o mesmo padrão de oclusão ou ser divididos em subgrupos iguais de tipos de oclusão.

Há certos tipos de oclusão que apresentam maior índice de reabsorção dentária do que outros. Ainda, conforme o tipo de oclusão, a intensidade e extensão do tratamento são menores ou maiores, o mesmo ocorrendo com as intensidades e tipos de forças aplicadas. Estes fatores mencionados podem interferir diretamente nos resultados observados, relativos a biopatologia da movimentação dentária induzida e das reabsorções dentárias.

\section{Padronização dos critérios, métodos e reali- zadores do diagnóstico e planejamento dos casos clínicos da amostra}

O diagnóstico e planejamento são mandatários para um bom tratamento ortodôntico e estes aspectos variam muito de acordo com o profissional e a sua forma de prognosticar. Varia inclusive conforme seu padrão de beleza, muito subjetivo. Assim, ao avaliar a movimentação dentária induzida e as reabsorções, na determinação da amostragem deve-se uniformizar:

a) Quem diagnosticou o paciente sob o ponto de vista ortodôntico? Foi o mesmo profissional ou vários? Se foram vários, como foi o processo de calibração que deve ser específico para cada trabalho?

b) Quem planejou os tratamentos? Foi o mesmo profissional ou vários? Como foi o processo de calibração que deve ser específico para cada trabalho? Os traçados cefalométricos são passíveis de variações?

c) Quem aplicou o tratamento? Foi o mesmo profissional ou vários? Como foi o processo de calibração que deve ser específico para cada tratamento?

\section{Uniformização e calibração dos operadores dos tratamentos}

Os operadores do tratamento possuem grande importância na uniformização da amostra, pois variam de acordo com o tempo de experiência de 
cada um: especialista ou aluno de especialização? Mestres ou doutores? Professores? As forças e a aplicação da técnica varia muito de acordo com o operador do tratamento! O paciente foi atendido sempre pelo mesmo profissional? Como foi a calibração destes operadores?

\section{Critério e métodos de diagnóstico das reab- sorções dentárias: radiografias utilizadas}

As radiografias utilizadas são periapicais e os dentes escolhidos quase sempre os incisivos superiores. Na metodologia deve-se cuidar de: como foram padronizados os aspectos de ângulo, distância, tipo de aparelhos, filmes e processamento? Houve calibração, foram sempre os mesmos? O técnico foi sempre o mesmo ou não? Se não, houve processo de calibração?

A escolha dos incisivos superiores como dentes alvos em pesquisas de reabsorção dentária em Ortodontia foi convencionada em que evento internacional de reconhecido valor científico? Outros dentes não são movimentados no tratamento ortodôntico? Será que os profissionais participantes da pesquisa não ficarão inconscientemente sugestionados a preservar ou atuar mais intensamente nos incisivos centrais de acordo com o seu interesse em obter-se um ou outro resultado do trabalho? Será que podemos desprezar resultados obtidos em outros dentes durante a realização do levantamento de resultados?

O diagnóstico de reabsorções dentárias em radiografias panorâmicas e telerradiografias em norma lateral (cefalométricas) é impreciso e questionável. Mesmo nas radiografias periapicais, as imagens das reabsorções apresentam limitações em sua interpretação, mas constituem ainda o melhor método de análise entre os acessiveis. Atualmente métodos tomográficos específicos para análise de imagens dentárias estão padronizados e disponíveis em alguns serviços no mundo, mas são ainda muito dispendiosos. Considerar válidos resultados obtidos em estudos de reabsorção dentária em Ortodontia a partir de telerradiografias ou panorâmicas implica em fragilizar as conclusões e parâmetros científicos.

Anamnese criteriosa: um dos segredos de prevenção de problemas quanto à reabsorção dentária em Ortodontia

Deve-se valorizar muito a anamnese inicial que deve ser criteriosa e muito bem dirigida para:

a) Traumatismo dentário prévio

b) Trauma oclusal

c) Onicofagia

d) Bruxismo

e) Interposição lingual

f) Outros vícios bucais

g) Tratamentos anteriores, mesmo que preventivos.

h) Contenções ativas

A cada consulta, uma anamnese dirigida deve ser realizada para detectar fatores importantes que possam ter atuado entre as duas últimas consultas.

\section{Análise da morfologia dentária e da crista ós- sea alveolar}

A morfologia dentária e da crista óssea alveolar deve ser considerada em todos os pacientes. Algumas formas de raiz e de crista óssea alveolar quando acometidas por reabsorções dentárias aparecem mais precocemente e intensamente que outros tipos morfológicos. Se isto não for considerado, os resultados sofrerão interferência intra e intergrupos. Se você quer detectar um fenômeno, seu efeito mais puro e isoladamente possível, deve-se uniformizar a amostra, inclusive morfologicamente.

\section{Tempo de tratamento e observação radiográ- fica da ocorrência das reabsorções dentárias durante o tratamento ortodôntico \\ O tempo de tratamento foi mais ou menos o mesmo? Deve-se relevar isto, pois maior tempo, mais forças, mais movimentos, maior probabilida- de de reabsorções dentárias e outros fenômenos}


acontecerem. Da mesma forma, o tempo de visualização e análise do caso após o tratamento deve ser uniforme.

Ainda sobre tempo, o momento da tomada radiográfica é importante no controle das reabsorções dentárias durante o tratamento ortodôntico. Houve a preocupação de uniformizar e escolher o tempo mais adequado para este controle?

\section{Técnica ortodôntica aplicada na amostra}

Quando da determinação do índice de reabsorção dentária em tratamento ortodôntico é muito comum a construção de grupos onde se incluem várias técnicas de tratamento e suas variáveis. Para serem validados, estes grupos devem ser uniformizados quanto às técnicas de tratamento ortodôntico e um mínimo de variáveis possíveis.

Dois exemplos podem ser utilizados: 1) Foram agrupados ou subgrupados os pacientes que usaram elásticos intermaxilares durante o tratamento? 2) Foram agrupados ou subgrupados os pacientes com extração ou sem extração dentária no tratamento ortodôntico?

\section{Consideração final}

Nas análises criteriosas sobre estudos referentes a Biopatologia da Movimentação Dentária Induzida e das Reabsorções Dentárias pode-se aplicar um questionário ou uma lista de checagem (checklist) na forma de um marcador de página, a cada item preenchido na metodologia do trabalho em leitura pode-se fazer uma marcação. No final da leitura, ter-se-ia um verdadeiro escore, placar de critérios preenchidos. Uma marca de que o trabatho realmente deve ser levado em consideração e utilizado como referência para os demais pesquisadores e profissionais clínicos em seus protocolos de investigação e de atuação clínica. Nesta lista, portanto, incluir-se-ia:

1) Estabelecimento de grupo controle negativo e positivo;

2) Uniformização da amostra quanto às características pessoais;
3) Padronização do tipo de má oclusão;

4) Padronização dos critérios, métodos de diagnóstico e planejamento;

5) Uniformização na aplicação dos procedimentos técnicos;

6) Calibração de operadores clínicos;

7) Padronização dos meios de diagnóstico: radiografias periapicais;

8) Anamnese criteriosa e uniformemente aplicada na amostra;

9) Análise morfológica da raiz dentária e da crista óssea alveolar;

10) Uniformização do período de tratamento;

11) Tempo de diagnóstico das reabsorções no tratamento;

12) Padronização da técnica ortodôntica aplicada.

Por fim, pode-se perguntar: existe na literatura algum trabalho perfeito do ponto de vista metodológico? A resposta é não, mas pode-se considerar muitos como relevantes e quase perfeitos: são aqueles que apresentaram e destacaram as limitações metodológicas, as explicitaram e as analisaram na interpretação, na discussão dos seus resultados e na elaboração de suas conclusões. 


\title{
About some methodological aspects of research in induced dental movement and of dental resorptions: a proposal of guide and cares for works analysis
}

\begin{abstract}
One of the main features of scientific methodology is the detailed description of all experimental conditions especially the used criteria whenever selecting a certain sample or obtaining data. This feature must be applied in every study so that another researcher can repeat the same work, under the same described conditions, and find the same results in any other part of the world. In addition, this procedure allows data checking and provides more credibility to the results. In science all data must be public and reproducible. Root resorption may be induced and influenced by several factors such as dental or alveolar crest morphology, crown-root proportion, type of malocclusion, orthodontic technique used, movement extensiveness and others. Therefore, standardizing the sample, detailing the diagnostic or classification criteria are essential whenever studying a multifatorial phenomenon, such as root resorption. In order to obtain credibility before the scientific community in a study about this subject a very homogeneous sample is required, as well as a strictly standardized diagnostic strategy and calibrated orthodontic technique. Unfortunately several papers on root resorption, especially concerning orthodontic treatment, are performed in accumulated samples without diagnostic or technique standardizing. Moreover, different clinicians not taking under consideration the need of sample uniformity of important variables usually treat the evaluated patients. In addition, most limitations are never mentioned, revealed or discussed when results are analyzed. The main goal of this paper is to induce some thinking about the subject and to suggest some parameters of analysis when evaluating research studies related to root resorption and biology of orthodontic movement.
\end{abstract}

Key words: Dental resorption. Root resorption. Orthodontic movement. Induced tooth movement. Methodology. Methodology of research.

\section{REFERÊNCIAS}

Se neste pequeno texto incluíssemos todos os trabalhos, monografias, dissertações e teses que tratam de cada um destes tópicos abordados teríamos uma lista imensa que teria pouca valia para o leitor. Em cada um dos trabalhos lidos, e que serviram de base para a construção deste texto, analisou-se a aplicação destes cuidados metodológicos ou não. São muitos trabalhos, alguns muito bons, outros nem tanto, mas que serviram do mesmo modo como objetos de análise. No livro sobre Reabsorções Dentárias nas Especialidades Clínicas apresenta-se um banco de referências com milhares de trabalhos referentes aos temas mencionados e que podem ser resgatados da literatura mundial.
1. ENCICLOPÉDIA Britânica on-line. Disponível em: < http:// www.britannica.com/ou http://www.eb.com/ >. Acesso em: 2 abr. 2004

2. BRUDVIK, P.; RYGH, P. The repair of orthodontic root resorption: an ultrastrutural study. Eur J Orthod, London, v. 17, no. 3, p. 189-198, June 1995.

3. CONSOLARO, A. Reabsorções dentárias nas especialidades clínicas. Maringá: Dental Press International, 2002.

Endereço para correspondência

Prof. Dr. Alberto Consolaro

Alameda Octávio Pinheiro Brisolla, 9-75 - Vila Universitária

CEP 17012 - 901 - Bauru - SP

alberto@fob.usp.br 